\title{
Suboptimal care of peanut allergic children in Germany concerning professional dietary advice and the supply of self-injectable epinephrine
}

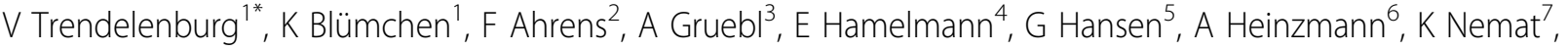 \\ B Niggemann ${ }^{8}$, K Beyer $^{1}$
}

From Food Allergy and Anaphylaxis Meeting (FAAM 2013)

Nice, France. 7-9 February 2013

\section{Background}

Patients with peanut allergy are advised to strictly avoid peanuts in their diet and to carry an emergency kit including self-injectable epinephrine at all times.

The aim of the study was to determine to which extent peanut allergic children in Germany are provided with self-injectable epinephrine and if they had received professional dietary advice.

\section{Methods}

63 peanut allergic children (median: age 6 years; peanutspecific IgE 73,5kU/l) who participated in an oral immunotherapy (OIT)-study were enrolled. Data concerning age of diagnosis, provision with self-injectable epinephrine, professional advice and performance of a peanut-free diet as well as the characterization of accidental allergic reactions to peanut (grade of severity I-V) were collected at their baseline visit of the OIT-study.

\section{Results}

At baseline visit, 17/63 children (27\%) had not been provided with self-injectable epinephrine although the diagnosis of their peanut allergy was already made 4 years (median) ago. Five of these 17 children had at least one severe allergic reaction after accidental peanut consumption (grade IV) in their history. 27/63 children (43\%) had not received self-injectable epinephrine at initial diagnosis but later in their life. In 13 of these 27 cases self-injectable epinephrine was prescribed only after at least one severe allergic reaction to peanut (grade IV). 19/63 children (30\%) had received self-injectable epinephrine directly at

Dept. of Pediatric Pneumology and Immunology, Charité University Medicine, Berlin, Germany

Full list of author information is available at the end of the article initial diagnosis. A strict avoidance of peanut was performed by $51 / 63$ families (82\%). 12/63 families (18\%) did not avoid products labelled with "may contain traces of peanuts". Only 13/63 families (21\%) had received dietary advice from a dietician.

\section{Conclusion}

Currently, peanut allergic children are not sufficiently provided with self-injectable epinephrine and do not receive enough professional dietary advice in Germany. Accidental allergic reactions are common. Therefore we recommend prescribing an emergency kit including self-injectable epinephrine directly at initial diagnosis. Furthermore families should receive an emergency training and a professional dietary advice.

\section{Disclosure of interest \\ None declared.}

\section{Author details}

${ }^{1}$ Dept. of Pediatric Pneumology and Immunology, Charité University Medicine, Berlin, Germany. ${ }^{2}$ Children's Hospital "Altona", Hamburg, Germany. ${ }^{3}$ Dept. of Pediatrics, Technical University Munich, Munich, Germany. ${ }^{4}$ Dept. of Pediatrics, Ruhr-University Bochum, Bochum, Germany. ${ }^{5}$ Dept. of Pediatric Pneumology, Allergology and Neonatology, Hannover Medical School, Hannover, Germany. ${ }^{6}$ Center for Pediatrics and Adolescent Medicine, University of Freiburg, Freiburg, Germany. ${ }^{7}$ Dept. of Pediatrics, University Hospital Carl Gustav Carus, Technical University of Dresden, Dresden, Germany. ${ }^{8}$ Pediatric Allergology and Pneumology, German Red Cross Hospital Westend, Berlin, Germany.

Published: 25 July 2013

doi:10.1186/2045-7022-3-S3-P38

Cite this article as: Trendelenburg et al: Suboptimal care of peanut allergic children in Germany concerning professional dietary advice and the supply of self-injectable epinephrine. Clinical and Translational Allergy 2013 3(Suppl 3):P38. 